\title{
ÉTUDE DE TROIS CAS \\ DE RHINOSPORIDIUM SEEBERI EN IRAN
}

\author{
Par HABIBI
}

L'existence du Rhinosporidium seeberi était inconnue en Iran jusqu'à 1937. J'ai eu l'occasion d'observer à Téhéran les deux premiers cas d'infection par ce parasite chez trois malades atteints d'une tumeur de la fosse nasale et dont nous rapportons ici les observations.

Etant donné le peu de temps qui s'est passé depuis que j'ai commencé à exercer la médecine à Téhéran (à partir de la fin de 1936) la rencontre de ces deux cas de tumeur parasitaire montre que cette maladie doit être assez fréquente en Iran.

Observation I. - En mars 1937, Dj. H., âgé de 23 ans, étudiant, consulte un confrère pour une gêne dans la respiration nasale, qui date depuis plusieurs mois. L'examen clinique montre une tumeur superficielle et saillante, située dans la région du cornet inférieur de la fosse nasale gauche.

La tumeur paraît être de la grosseur d'une noisette, collée sur la face interne du cornet, non pédiculée, d'un aspect polypomateux, lisse et pâle. Une biopsie est envoyée au Laboratoire Central de l'Hygiène Publique pour vérifier la nature bénigne ou maligne de la tumeur.

L’étude histologique du fragment prélevé a montré la présence du Rhinosporidium seeberi dans l'épaisseur de la muqueuse nasale et en même temps une réaction inflammatoire un peu particulière, ressemblant à celle que l'on trouve dans les tumeurs inflammatoires dues à des corps étrangers et non un processus tumoral proprement dit.

Pendant que nous étions en train de faire l'inclusion de la pièce, le médecin traitant, pour soulager son malade, avait enlevé la tumeur tout entière (pesant 15 grammes environ) qu'il nous envoya de nouveau. Sur ce morceau nous avons fait des coupes sériées pour étudier l'évolution du parasite, ainsi que les lésions anatomo-pathologiques de la muqueuse nasale.

Evolution du parasite. - Cette évolution comprend morphologiquement et un peu arbitrairement trois sortes d'éléments :

Andales de Parasitologie, T. XVII, x². $-1^{\text {er }}$ mars 1939 , p. 103-107. 
a. Petits éléments de 5 à $20 \mu$ à protoplasma vacuolaire et rétracté, à noyau clair avec un nucléole central (pl. VI, fig. 4).

b. Moyens éléments de 20 à $100 \mu$ dont la paroi est formée d'une membrane anhiste, épaisse, colorée en rose par l'hématéine éosine ou l'érythrosine et en bleu et vert par le bleu de Masson et le vert lumière. Leur cytoplasme est clair, rétracté et vacuolaire, le noyau assez gros et plus visible (pl. VI, fig. 4).

c. Alors que les deux formes précédentes étaient précoces les gros éléments sont des formations tardives, ayant parfois $200 \mu$ de diamètre. Leur membrane est épaisse, d'aspect cellulosique brillant, renfermant des centaines d'éléments arrondis ou ovalaires, noyés dans une substance mucoïde (pl. VI, fig. 4).

L'étude des coupes sériées de ces sporanges est très intéressante car elle nous montre :

1. Dans une première phase l'apparition d'un petit pore d'une dizaine de $\mu$ de diamètre sur la paroi externe des sporanges par lequel les spores s'échappent mais toujours en petit nombre (pl. V, fig. 1, 2, 4).

L'existence de ce pore, bien que S.-E. Parodi (1926) la nie, est constante et ces pores sont toujours entourés d'un bourrelet nettement visible sur la paroí externe du parasite dans les moyens et gros éléments (pl. VI, fig. 4) ; il est absent sur les petits éléments, car l'ébauche commence sur les éléments de moyenne grosseur avant que le pore se forme (pl. V, fig. 1 et 2).

2. Dans sa seconde phase, qui suit toujours la précédente, il y a un éclatement brusque de la paroi qui est tout à fait renversée se vidant d'un coup de son contenu, comme si le pore était insuffisant à évacuer le trop plein. L'augmentation de la pression intérieure et la diminution de la résistance de la paroi font crever l'enveloppe à son point faible qui est le pore : d'ailleurs l'échappement en fusée des spores est démonstratif (pl. VI, fig. 1 et 2).

Disons également que nous avons trouvé les trois sortes d'éléments presque partout, aussi bien dans l'épaisseur qu'à la surface de la muqueuse nasale. Leur présence superficielle nous incita à les chercher dans la sécrétion nasale, oủ nous avons trouvé quantités de spores et même de sporanges mûrs (pl. VI, fig. 3).

Lésions anatomo-pathologiques. - Au point de vue anatomopathologique, nous trouvons une réaction inflammatoire macrophagique diffuse et des lésions folliculaires nettes, superposables aux lésions causées par les autres champignons. Cependant il 
existe en apparence deux sortes de lésions qui sont en rapport avec des diverses périodes de l'évolution des parasites.

1. Les lésions folliculaires qui comprennent: une zone centrale. formée souvent par les polynucléaires plus ou moins altérés et par des parasites en général mûrs ; une zone moyenne, formée par des cellules épithélioïdes et géantes et une zone périphérique sclérosée, infiltrée par des cellules inflammatoires banales (pl. V, fig. 3 et pl. VI, fig. 1 et 2 ).

2. Des réactions inflammatoires macrophagiques diffuses, sans aspect folliculaire net et ceci aux endroits où les parasites jeunes sont très nombreux (pl. VI, fig. 4).

Ces lésions anatomo-pathologiques peuvent être plus ou moins marquées suivant les cas : dans notre première observation, par exemple, elles sont beaucoup plus accusées que dans la seconde.

ObServation II. - En juin 1938, A., âgé de 76 ans, commerçant en thé qui n'a jamais quitté l'Iran, souffrant depuis presqu'un an de troubles de la respiration nasale caractérisés par des rhumes fréquents, des douleurs irradiantes dans les oreilles, des saignements de nez, consulte le service d'oto-rhino-laryngologie de l'hôpital Dowlati. A l'examen, on trouve une petite tumeur dans la fosse nasale droite; une biopsie de cette tumeur nous est envoyée pour faire un examen histologique. La tumeur présente une consistance molle, friable; elle est de teinte rose pâle, d'aspect framboisé et saigne très facilement au toucher. L'autre cavité nasale est restée cependant indemne. Rien à signaler dans l'entourage du malade, la source de la contagion nous échappe. Le malade est scus notre surveillance médicale (solution d'émétique de 2/100 d'après Wright).

A l'examen histologique, nous avons trouvé encore le Rhinosporidium seeberi avec les mêmes constatations microscopiques que dans le cas précédent, seulement les lésions anatomo-pathologiques sont beaucoup moins marquées.

Au point de vue clinique notons : la tumeur était préalablement implantée sur la partie inférieure et tout à fait en avant de la cloison ; à quelques millimètres de la narine droite, on apercevait, sur la paroi externe de la même cavité nasale, d'autres petites tumeurs isolées, d'évolution récente et de mème nature, aussi bien au point de vue de l'aspect clinique que de l'examen histologique, ce qui prouve l'absence de l'immunité locale, et une contagion directe par les spores ou par des greffes du tissu tumoral sur les parties saines. 
Observation III. - Mlle R., fillette de 10 ans, habitant dans un petit village de l'Azarbaïedjan, consulte le $\mathrm{D}^{\mathrm{r}}$ Alavi pour une tumeur de la fosse nasale gauche qui sortait presque de sa narine.

A l'examen on a signalé l'existence de deux tumeurs polypomateuses, molles, friables, saignant facilement, fixées l'une sur la partie inférieure et l'autre sur la partie supérieure de la cloison.

L'histoire de la maladie remonte à deux ans ; on n'a pas signalé de cas chez ses parents et dans son entourage, aussi la source de contagion nous échappe.

Cependant un point suspect est que l'enfant joue souvent avec des moutons, des chèvres, etc. Le médecin traitant enlève la tumeur et nous en envoie un petit fragment pour faire un examen histologique. Diagnostic histologique : Rhinosporidium seeberi.

\section{EXPLICATION DES PLANCHES V et VI}

\section{Planche $V$}

\section{Rhinosporidium seeberi}

FIG. 1. - Sporange mûr avec pore périphérique entouré par un bourrelet.

Fig. 2, - Sporange mûr laissant échapper des spores par son pore. Ce sporange est entouré de leucocytes polynucléaires (première observation).

FIc. 3. - Follicules multiples à cellules épithélioïdes et géantes en train de phagocyter les parasites.

Fig. 4. - Deux sporanges: l'un mûr, avec pore périphérique et bourrelet; l'autre avec bourrelet bien visible coupé perpendiculairement et dont la partie creusée donnera le futur pore périphérique.

\section{Planche VI}

Fic. 1. - Un sporange au moment de sa rupture. Autour du sporange une masse de polynucléaires entourée de cellules épithélioïdes.

Fig. 2. - Sporange complètement vidé et retourné. Au centre du follicule on voit une masse de polynucléaires entourés de cellules épithéliö̈des encerclées de bandes sclérosées.

Fig. 3. - Spores dans la mucosité nasale.

Fig. 4. - Coupe de la tumeur du deuxième malade vue à un faible grossissement et montrant les divers stades des éléments parasitaires. 
ANNALES DE PARASITOLOGIE

T. XVII, No 2, 1er Mars 1939
Planche V

(Mémoire HaBıBI)

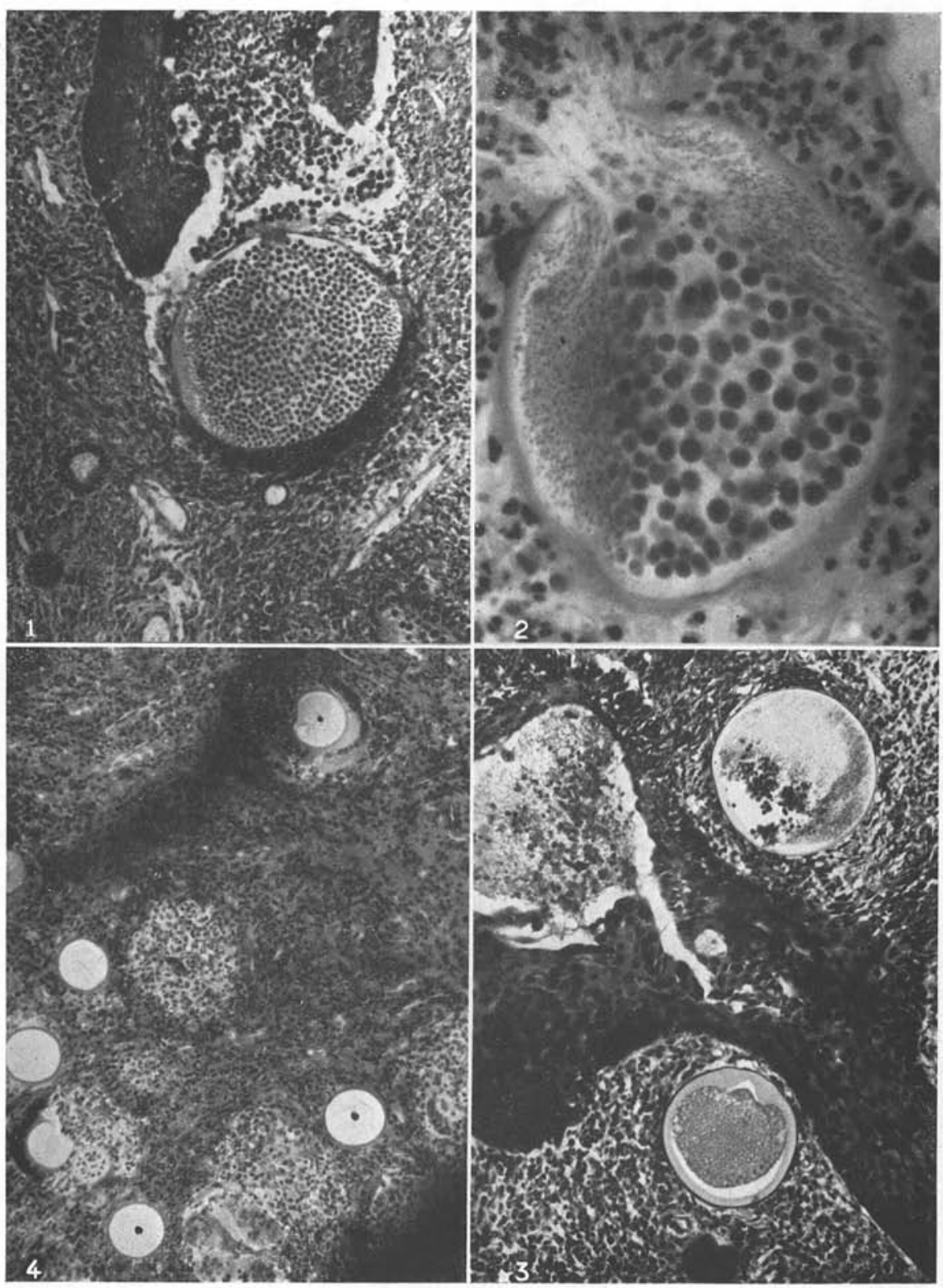


ANNALES DE PARASITOLOGIE

T. XVII, No 2, 1er Mars 1939
Planche VI

(Mémoire НАBıBI)

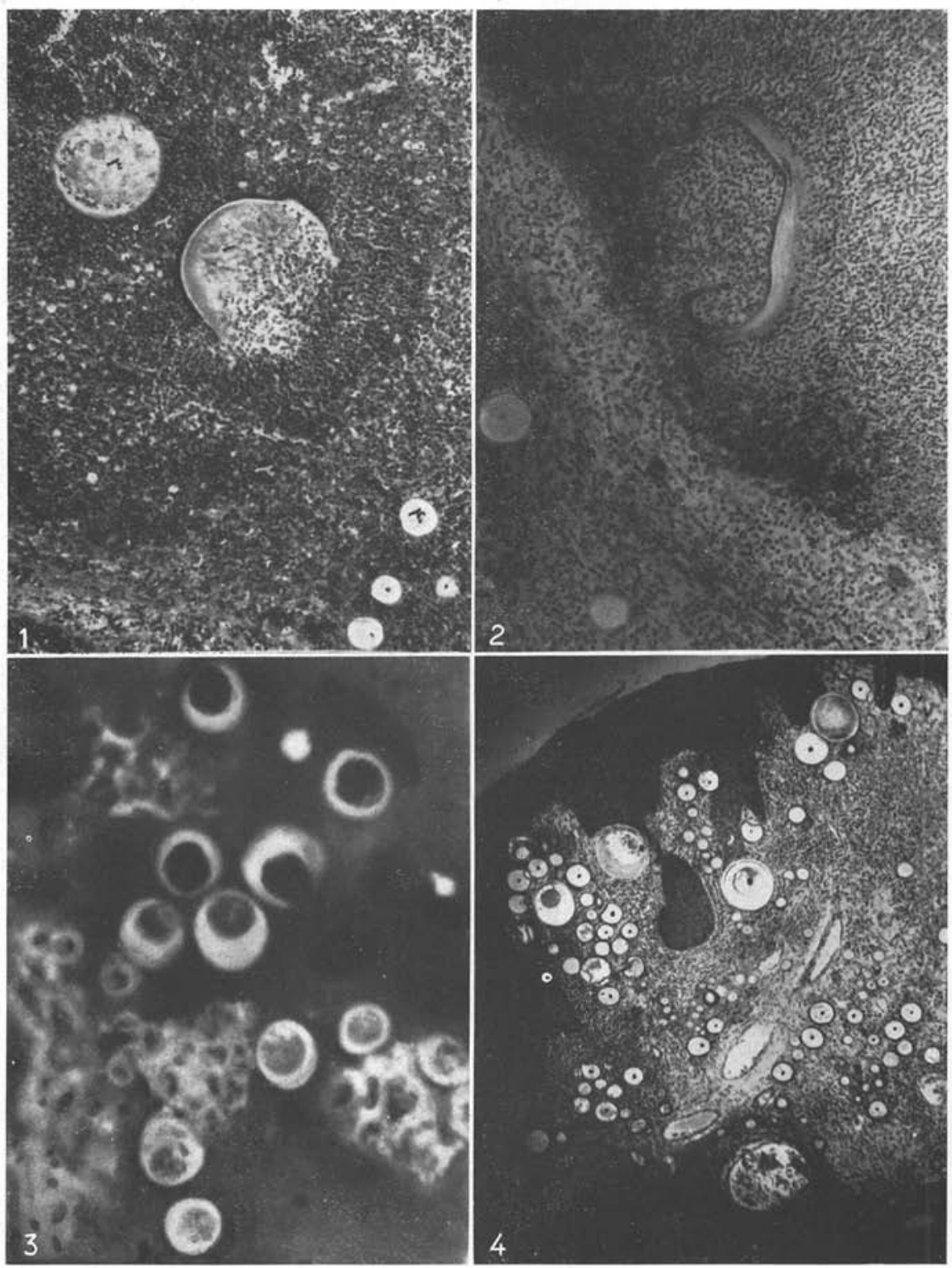




\section{RÉSUMÉ}

1. Présence constante d'un pore dans les sporanges, quand ils arrivent à une certaine période de leur évolution.

2. Présence d'un bourrelet périphérique sur la paroi du sporange, bien visible même sur les sporanges encore non mûrs.

3. Eclatement brusque des sporanges quand ils arrivent à une certaine période de leur évolution, à cause de l'insuffisance de l'ouverture du pore pour l'échappement des spores et dẻ l'augmentation de la pression intérieure.

4. Existence des spores dans la sécrétion nasale qui est probablement la source de contagion.

5. Lésions anatomo-pathologiques microscopiques correspondant à une réaction inflammatoire macrophagique avec foyers nodulaires.

6. Fréquence en Iran.

Laboratoire d'anatomie pathologique de la Faculté de médecine de Téhéran. 